\title{
IDENTIDAD DE TIPOS Y HACEDORES DE VERDAD
}

JOAN Pagès

Departament de Filologia i Filosofia

Universitat de Girona joan.pages@udg.es

SUMMARY: In this paper I deal with Armstrong's last theory of states of affairs and its relation to truthmakers for sentences and the problem of universals. More specifically, I discuss his truthmaker principle, rejecting some of the objections that has been raised against it. However, I also try to show that Armstrong's answer to the problem of the negative existencial sentences in terms of totality states of affairs is mistaken. Finally, I rebut Oliver's slinghot argument against truthmakers and also discuss Armstrong theory of states of affairs as truthmakers.

KEY WORDS: universals, truthmakers, states of affairs

RESUMEN: Este artículo trata de la última teoría de los estados de cosas de Armstrong, así como de su relación con los hacedores de verdad de los enunciados y con el problema de los universales. En particular, se discute su principio del hacedor de verdad, y se rechazan algunas de las objeciones que se han presentado al mismo. Por otra parte, se intenta mostrar también que la respuesta de Armstrong al problema de los enunciados existenciales negativos en términos de estados de cosas de totalidad es errónea. Finalmente, también se objeta la versión de Oliver del argumento de la honda y se discute la teoría de los hacedores de verdad como los estados de cosas.

PALABRAS CLAVE: universales, hacedores de verdad, estados de cosas

Una de las cuestiones más debatidas en filosofía por su centralidad es la concerniente a la existencia de los universales. Entre los diversos y variados filósofos actuales que han abordado esta compleja cuestión, David Armstrong ha sido y continúa siendo uno de los autores que han defendido una posición realista al respecto. Para Armstrong, es necesario postular la existencia de universales en una teoría de las propiedades aceptable. A su juicio, tres son las razones principales que abogan por esta necesidad: el compromiso ontológico de enunciados con términos singulares 
abstractos y cuantificadores de propiedades, el análisis de las leyes naturales y la causalidad, y el problema de la identidad de tipos. Sin embargo, el fundamento y alcance de sus argumentos no resultan del todo claros y merecen, sin duda, una revisión crítica que contribuya a su evaluación. Un reciente artículo de Alex Oliver $^{1}$ ha contribuido considerablemente a esclarecer la posición de Armstrong, al proponer diversas líneas interpretativas que permiten dar cuenta de sus argumentos y evaluarlos con mayor facilidad. Nuestro propósito en este artículo es ahondar en la tarea iniciada en el artículo de Oliver, centrándonos en el problema de la identidad de tipos.

De acuerdo con Oliver, dicho problema puede entenderse de cuatro modos distintos y relacionados, respectivamente, con el análisis de la predicación, con el análisis de semejanza, con el criterio de compromiso ontológico y con la cuestión de los hacedores de verdad. ${ }^{2}$ De acuerdo con la evidencia textual previa a la publicación de su artículo, Oliver sostiene que la interpretación del problema de la identidad de tipos que resulta más razonable atribuir a Armstrong es la que involucra el criterio de compromiso ontológico. Sin embargo, en el último libro de Armstrong, la noción de hacedor de verdad adquiere una relevancia fundamental, y también hallamos algunos pasajes que sugieren que la versión del problema que le preocupa es la vinculada a los hacedores de verdad. Por todo ello, y porque, aun sin estar exenta de dificultades, nos parece la interpretación más caritativa, así como la menos explotada, nuestra discusión se centrará en esta cuarta interpretación posible del problema.

Antes de entrar a comentar las dificultades que se derivan de cada una de las interpretaciones del problema de la identidad de tipos propuestas, conviene aclarar de dónde surgen. Puede decirse que el problema de la identidad de tipos es el problema de dar una explicación a un tipo de hechos. Las diversas posibilidades interpretativas acerca del problema surgen de las alternativas que

1 Oliver 1996.

${ }^{2}$ La expresión 'hacedor de verdad' pretende traducir la expresión inglesa 'truthmaker'. 
existen en relación con el tipo de explicación y con el tipo de hechos involucrados. En este sentido, Oliver distingue tres tipos de hechos estrechamente vinculados. En primer lugar, tenemos los hechos de tipo A, consistentes en que un particular ejemplifica una propiedad (p.ej.: el hecho consistente en que cierta manzana ejemplifica la propiedad de tener 150 g. de masa). En segundo lugar, tenemos los hechos de tipo B, consistentes en que dos particulares ejemplifican cierta propiedad común (p.ej.: el hecho consistente en que dos manzanas ejemplifican la propiedad de tener $150 \mathrm{~g}$. de masa). En tercero y último lugar, tenemos los hechos de tipo C, consistentes en que dos particulares se asemejan en cierto aspecto (p.ej.: el hecho consistente en que dos manzanas se asemejan en el aspecto masa).

Así, Lewis y Van Cleve interpretan que Armstrong se está ocupando de hechos de tipo A, de forma que una solución al problema de la identidad de tipos, así entendido, exigiría un análisis de la predicación. ${ }^{3}$ Por otra parte, Mortensen cree que el problema de la identidad de tipos, tal como lo entiende Armstrong, está conectado directamente con las perspectivas de ofrecer un análisis de la semejanza entre particulares. ${ }^{4}$ Devitt y Oliver identifican el problema de la identidad de tipos presentado por Armstrong con la aplicación del criterio del compromiso ontológico a enunciados que expresan hechos de tipo A. ${ }^{5}$ En la primera sección de este artículo comentaremos brevemente estas propuestas interpretativas y los problemas que suponen para la posición de Armstrong. Asimismo, Oliver también considera la hipótesis de que el problema de la identidad de tipos esté relacionado con aquello que hace verdaderos a esos enunciados (esto

3 Lewis 1983 y Van Cleve 1994.

4 Mortensen 1987.

${ }^{5}$ Devitt 1980 y Oliver 1996. Oliver, que es el primero en hacer explícitos los problemas que existen para entender con precisión qué problema está presentando Armstrong, es también el primero en reconocer que no está claro cómo debemos interpretar la evidencia textual. A pesar de que el resto de alternativas interpretativas hallan también algún fundamento en las afirmaciones de Armstrong, probablemente Oliver está en lo cierto cuando sostiene que la interpretación que mejor encaja con la mayoría de las afirmaciones de Armstrong es la de Devitt. 
es, el hacedor de verdad de ellos) que expresan hechos del tipo B. Esta línea de interpretación será nuestro objeto de análisis en la segunda sección de este artículo.

1. Análisis de la predicación, análisis de semejanza y compromiso ontológico

\subsection{Análisis de la predicación}

Como ya hemos apuntado, Lewis y Van Cleve entienden que Armstrong está exigiendo un análisis de la predicación que se aplique a todas las instancias predicativas del tipo 'a es F', pero generalizada a predicación de cualquier ariedad. A juicio de Armstrong las tentativas nominalistas de análisis fracasan en su intento de dar cuenta del problema de la identidad de tipos así interpretado. Sin embargo, si el argumento de Armstrong a favor de los universales se basa en el problema de la identidad de tipos entendido como análisis de la predicación, entonces, como Van Cleve y Lewis han mostrado, se trata de un argumento fallido. La fuerte exigencia del análisis predicativo requiere de una teoría de los universales el postulado de la relación de ejemplificación, el cual comporta la aparición de una regresión (Lewis 1983) y genera un análisis que invierte el orden natural de la explicación (Van Cleve 1994) y que, además, deviene necesariamente incompleto (Van Cleve 1994).

\subsection{Análisis de semejanza}

Una segunda interpretación del problema de la identidad de tipos presentado por Armstrong surge al concebirlo como una demanda de explicación, entendida como un análisis conceptual, de los hechos de tipo C. Es obvio que podría elaborarse una explicación natural de estos hechos en términos de los hechos de tipo B, relativos a dos particulares que ejemplifican la misma propiedad: asemejarse en el mismo aspecto puede explicarse de modo natural en términos de compartir una propiedad.

La idea de Armstrong es sacar partido de estas conexiones conceptuales entre semejanzas y propiedades, así como de su teoría de las propiedades que identifica a éstas con universales, de forma que el postulado de universales permita analizar los 
predicados básicos de semejanza. Así, se contempla que dos particulares se asemejan (en cierto aspecto) si y sólo si ejemplifican un mismo universal o ejemplifican universales semejantes. ${ }^{6}$

No obstante, como Oliver y Mortensen indican, el argumento de Armstrong a favor de los universales basado en consideraciones de semejanza no está exento de serias dificultades. Aun aceptando la tesis de que, en general, los hechos de semejanza deben ser analizados, existen casos en que claramente el análisis no es prescriptivo (Mortensen 1987). Además, si dicho análisis debe ser implementado por una teoría de los universales que incorpore la relación de ejemplificación, entonces se genera una regresión (Oliver 1996).

\subsection{Compromiso ontológico}

En la tercera interpretación distinguida por Oliver, el problema de la identidad de tipos se concibe como un problema que afecta en primera instancia a los hechos de tipo B. Sin embargo, la formulación correcta de esta versión del problema, en realidad, debe efectuarse en términos parcialmente lingüísticos y no directamente ontológicos como acabamos de hacer: se trata de establecer el compromiso ontológico de los enunciados que expresan la identidad de tipos entre particulares, o, simplemente, el de los enunciados predicativos. ${ }^{7}$ De esta forma, la investigación del compromiso ontológico de los enunciados que expresan

${ }^{6}$ Armstrong 1978, II, p. 96. Como se puede observar, el análisis no permite desprenderse en primera instancia de todos los posibles predicados de semejanza, pues todavía aparece un predicado de semejanza en el analisans que ahora se aplica a universales, en lugar de aplicarse a particulares. Sin embargo, Armstrong ofrece un análisis de este último predicado de semejanza en términos de identidad parcial. Pensemos en magnitudes como tener $3 \mathrm{~kg}$ de masa, o tener $6 \mathrm{~kg}$ de masa. Según Armstrong, estos universales de masa son parcialmente idénticos; de hecho, el primero es parte del segundo. El grado de semejanza entre dos magnitudes cualesquiera de un mismo tipo se explica entonces por la proporción entre la parte común a ambos universales respecto de la parte no común.

7 Devitt (1980) explicita cómo el argumento de Armstrong asume la equivalencia entre cualquier enunciado que expresa identidad de tipo (e.g. ' $a$ y $b$ ejemplifican la F-idad') con la conjunción de enunciados predicativos simples (' $a$ es F' y ' $b$ es G'). 
la identidad de tipos entre particulares pasaría por la investigación del compromiso ontológico de los enunciados predicativos en general. Sin embargo, como Oliver (1996) convincentemente muestra, el argumento de Armstrong basado en consideraciones de compromiso ontológico es incompatible con su realismo $a$ posteriori y, además, la aplicación de dicho criterio bajo sus propios supuestos genera regresiones del mismo tipo que Armstrong aduce para rechazar el tratamiento nominalista del problema.

\section{Hacedores de verdad}

La última interpretación ensayada por Oliver en relación con el problema de la identidad de tipos, tal como lo presenta Armstrong, pone en juego la noción de hacedor de verdad. Según esta interpretación, Armstrong estaría argumentando que la mejor teoría de los hacedores de verdad de los enunciados que expresan la identidad de tipos, y afines, requiere el postulado de universales. ${ }^{8}$ De acuerdo con Armstrong, los hacedores de verdad de los enunciados descriptivos son estados de cosas, los cuales involucran universales. Aunque si se consideran los trabajos de Armstrong en los que se basan las conclusiones de Oliver, no puede decirse que la interpretación del problema de la identidad de tipos en términos de hacedores de verdad sea la más ajustada, lo cierto es que el último libro de Armstrong, posterior al artículo de Oliver, abona esta interpretación. En este libro, Armstrong otorga un papel fundamental a la noción de hacedor de verdad y trata de elucidarla, a diferencia de lo que puede observarse en trabajos anteriores.

Nuestro plan para esta sección es el siguiente. En primer lugar, trataremos de dar cuenta de la noción de hacedor de verdad. En segundo lugar, presentaremos un principio asociado a esta noción y cuatro objeciones que diversos autores han formulado a este principio, así como nuestra réplica a tres de las mismas. En tercer lugar, expondremos y criticaremos la defensa que Armstrong realiza del principio apelando a estados de cosas de totalidad. En cuarto lugar, comentaremos el alcance del argumento

8 Oliver 1996, p. 68. 
de la honda para los hacedores de verdad. Finalmente, nos centraremos en la teoría de los estados de cosas como hacedores de verdad, que, a nuestro juicio, entraña las dificultades más importantes para el realismo de universales.

\subsection{La noción de hacedor de verdad}

La idea de Armstrong es que un hacedor de verdad es aquello en el mundo que hace verdadera una verdad. ${ }^{9}$ Esta noción de Armstrong había sido descrita antes por Mulligan, Simons y Smith, ${ }^{10}$ por un lado, y por Fox ${ }^{11}$ por el otro. Así, los primeros autores mencionados caracterizan la noción de hacedor de verdad en el párrafo siguiente:

A lo largo del auge realista de los primeros años de este siglo, filósofos de diversas tendencias se ocuparon de investigar la ontología de la verdad. Esto es, concibiesen o no la verdad como correspondencia, estaban interesados en el grado de necesidad que hay de suponer la existencia de entidades que ejercieran algún papel en la explicación de la verdad de los enunciados [...] Sin embargo, algunos pensadores como Russell, el Wittgenstein del Tractatus y el Husserl de las Logische Untersuchungen, argumentaron que en lugar de, o además de, portadores de verdad, hay que suponer la existencia de ciertas entidades en virtud de las cuales los enunciados y/o las proposiciones son verdaderas [...] llamaremos hacedores de verdad a cualesquiera entidades que sean candidatas a este papel. ${ }^{12}$

La caracterización de Fox es más somera: "por hacedor de verdad para $A$, entiendo algo cuya existencia implica $A$ ". ${ }^{13} \mathrm{~A}$ juicio de Armstrong, el tipo de entidades que satisfacen el rol

9 Armstrong 1997, p. 13.

10 Mulligan, Simons y Smith 1984.

11 Fox 1987.

12 Mulligan, Simons y Smith 1984, pp. 287-288.

13 Fox 1987, p. 189. Oliver (1996, p. 69) define la noción en los mismos términos: "Así, podemos definir la noción relacional de un hacedor de verdad, $\mathrm{T}$, para un enunciado dado, S, como:

$\mathrm{T}$ es un hacedor de verdad para $\mathrm{S}$ si y sólo si 'T existe' implica ' $\mathrm{S}$ es verdadero'. 
descrito para los hacedores de verdad son los estados de cosas del mundo, entidades complejas que tienen dos tipos de constituyentes: particulares y universales.

Observemos que esta noción de hacedor de verdad asociado a un enunciado, que Fox define, no parece en primera instancia aplicarse de manera natural a las verdades necesarias. Toda entidad existente resulta ser, en la definición de Fox, un hacedor de verdad de cualquier verdad necesaria. Aunque Armstrong no ofrece la misma definición, sus estados de cosas son concebidos en primer lugar como hacedores de verdad específicos de verdades contingentes, mientras que el estatuto de los hacedores de verdad de las verdades necesarias no está claro en su teoría. ${ }^{14}$ Por otro lado, para Armstrong, la relación entre un hacedor de verdad de cierto enunciado verdadero y el enunciado verdadero mismo es, fijado el lenguaje, interna. ${ }^{15}$ Es decir, si T es un hacedor de verdad de $\mathrm{S}$ en el mundo actual, entonces es necesario que si $\mathrm{T}$ existe, entonces $\mathrm{S}$ sea verdadero. ${ }^{16}$

Observemos también que hasta el momento presente no hemos hablado de "el" hacedor de verdad de un enunciado. La razón de ello es que no todo enunciado tiene un hacedor de verdad único. Por ejemplo, 'Hay al menos un planeta que contiene oxígeno' tiene tantos hacedores de verdad como planetas con oxígeno. Por otro lado, no todo hacedor de verdad es un hacedor de verdad de un solo enunciado verdadero. Así, el estado de cosas consistente en que Pedro acompañara a Asunción al foniatra es un hacedor de verdad de los siguientes enunciados verdaderos: 'Pedro acompañó a Asunción al foniatra' y 'Pedro acompañó a Asunción al foniatra o Asunción llevó a Carla al concierto de rock'.

En realidad, incluso las definiciones de Fox y Oliver, más precisas y cuidadosas, presentan ciertos problemas que ellos mismos

14 Armstrong 1997, p. 2. Lo que sí parece claro es que las verdades necesarias carecen de hacedores de verdad específicos.

15 Ibid., p. 115.

16 De modo que la condición a partir de la cual Fox y Oliver definen la noción de hacedor de verdad resulta ser, para Armstrong, cuando menos necesaria. 
intentan resolver. Por ejemplo, dada la definición de hacedor de verdad, el mundo resultaría ser un hacedor de verdad de cualquier verdad, pues, dada una verdad cualquiera, la existencia del mundo implica su verdad. Fox y Oliver proponen ciertas modificaciones en la definición de hacedor de verdad para salvar este problema. ${ }^{17}$ Así, ambos autores identifican la noción buscada de hacedor de verdad con lo que denominan "hacedor de verdad mínimo": un hacedor de verdad mínimo de una verdad cualquiera es un hacedor de esta verdad ninguna de cuyas partes propias resulta ser un hacedor de verdad de la verdad en cuestión. (Observemos que este refinamiento no garantiza todavía que las asociaciones entre verdades y hacedores de verdad (y las opuestas) vayan de una en una, pues el enunciado 'Hay al menos un planeta con oxígeno' continúa teniendo tantos hacedores de verdad como planetas con oxígeno. Sin embargo, este aspecto no parece comportar ningún problema fundamental.)

\subsection{El axioma del hacedor de verdad}

Otra cuestión central concierne a lo que Fox denomina "axioma del hacedor de verdad"; se trata de la validez del principio que afirma que toda verdad tiene (al menos) un hacedor de verdad. Armstrong acepta el axioma, aparentemente sin restricciones, ${ }^{18}$ sosteniendo que resulta obvio cuando es objeto de examen. ${ }^{19}$ Los casos de enunciados existenciales negativos proporcionan, en primera instancia, contraejemplos claros al principio. ${ }^{20} \mathrm{En}$ mi opinión, estos casos recomiendan adoptar una versión restringida del axioma a los enunciados afirmativos. En el próximo apartado daremos cuenta del intento de Armstrong de salvar

17 Fox 1987, p. 190, Oliver 1996, p. 70.

18 A excepción, tal vez, de restricciones relativas al carácter modal del enunciado cuyo hacedor de verdad esté en cuestión. Sin embargo, no es clara su posición con relación a las verdades necesarias y la ausencia de hacedores de verdad específicos para las mismas.

19 Armstrong 1989, p. 89. En Armstrong 1997 usa el axioma en el argumento a favor de los estados de cosas sin aportar la menor justificación.

${ }^{20}$ Mulligan, Simons y Smith, 1984, p. 314. Véase también Lewis 1992, p. 216. 
algunos casos de existenciales negativos, a nuestro juicio fallido. En este apartado discutiré tres objeciones a la versión restringida del principio.

En primer lugar, Mulligan, Simons y Smith proponen dos tipos de contraejemplos a la versión del principio restringida a enunciados descriptivos simples. Su argumento es el siguiente:

Un enunciado simple como 'Pedro tiene hepatitis' puede ser verdadero aunque no haya ningún único objeto que lo haga verdadero: desde el punto de vista de sus hacedores de verdad, el enunciado puede comportarse como una conjunción no degenerada. Sin embargo, por lo que respecta, digamos, a 'Pedro busca a Asunción y Asunción busca a Carla' o 'Ha habido cuarenta presidentes de los Estados Unidos hasta 1981', resulta con seguridad contraintuitivo suponer que hay objetos únicos compuestos que hacen verdaderos a estos enunciados: un Pedro buscando a Asunción y un Asunción buscando a Carla mereológicamente unidos, o una fusión mereológica de todos los presidentes de los Estados Unidos desde Washington a Reagan (en la que a Grover Cleveland se le cuenta misteriosamente dos veces). ${ }^{21}$

A nuestro juicio, estas razones no parecen muy concluyentes. Primero, la necesidad de postular estados de cosas mereológicamente compuestos, como el hacedor de verdad del primer ejemplo, no nos parece contraintuitivo. La fusión de dos estados de cosas es un fragmento discontinuo del mundo que puede hacer verdadero un enunciado. Segundo, por lo que respecta al caso de los presidentes, su hacedor de verdad no es el agregado de los presidentes, sino la fusión mereológica de los estados de cosas consistentes en que cada uno de esos individuos son presidentes. El aparente misterio de "contar dos veces a Cleveland" desaparece, pues en lugar de Cleveland, el hacedor de verdad del enunciado contiene dos estados de cosas distintos que comparten el mismo particular.

${ }^{21}$ Mulligan, Simons y Smith, 1984, p. 313. En este mismo artículo (pp. 311-318), estos tres autores consideran y discuten toda una serie de principios putativos para los hacedores de verdad que constituyen una buena aproximación a una elucidación más profunda del concepto que, a buen seguro, queda todavía pendiente. 
En segundo lugar, Oliver observa que existen enunciados contingentes verdaderos, estructuralmente simples, cuyos hacedores de verdad no parecen ser el tipo de entidades a las que Armstrong atribuye el papel de hacedores de verdad. Así, enunciados existenciales como 'Sócrates existe', o enunciados de identidad como 'Sócrates es Sócrates', no parecen requerir estados de cosas que los hagan verdaderos. Sin embargo, entiendo que es posible dar cuenta de los referentes de los términos singulares que aparecen en enunciados como los mencionados en términos de estados de cosas: particulares con sus propiedades, de modo que el enunciado acerca de la existencia de Sócrates sería hecho verdadero por cualquiera de los estados de cosas que contienen a Sócrates ejemplificando alguna propiedad. ${ }^{22}$

A falta de contraejemplos oportunos, podemos concluir que la versión del axioma del hacedor de verdad restringida a verdades afirmativas contingentes resulta razonable.

2.3. La defensa de Armstrong del axioma del hacedor de verdad: estados de cosas de totalidad

En sus últimos trabajos, Armstrong incluye en su ontología lo que denomina "estados de cosas de totalidad" para explicar ciertos casos especiales de existenciales negativos. ${ }^{23}$ Consideremos la totalidad de los estados de cosas del mundo (T). Observemos que es verdad que todos estos estados de cosas son los únicos estados de cosas del mundo. Esta verdad puede expresarse mediante un existencial negativo, a saber:

(N) No existe ningún estado de cosas distinto de los que constituyen la totalidad $\mathrm{T}$.

El razonamiento de Armstrong para postular estados de cosas de totalidad parte de la necesidad de hallar un hacedor de verdad para $(\mathrm{N}) .{ }^{24} \mathrm{El}$ estado de cosas de totalidad es simplemente una

22 Nótese que si nuestro tratamiento de los enunciados de identidad es correcto, entonces hay hacedores de verdad para algunas verdades necesarias.

23 Armstrong 1997, cap. 13. Sin embargo, una solución para estos casos especiales puede extenderse fácilmente a todos los casos de existenciales negativos.

24 Armstrong 1997, p. 197. 
entidad introducida para satisfacer este rol de servir de hacedor de verdad de la verdad mencionada; es por ello que debe ser concebida como un hecho de clausura, o, simplemente, como el estado de cosas consistente en que todos los otros estados de cosas son los únicos estados de cosas del mundo. ${ }^{25}$

Armstrong argumenta a favor de la existencia de los estados de cosas de totalidad del siguiente modo:

Si es verdad que cierta conjunción de estados de cosas es la totalidad de los estados de cosas, entonces esto es verdad sólo porque no hay más estados de cosas. Si hay más, entonces la proposición no es verdadera. Que no hay más debe ser incoporado de algún modo en el hacedor de verdad. Pero afirmar que no hay más estados de cosas es decir que aquéllos son todos los estados de cosas. Esto, por tanto, debe ser incorporado en el hacedor de verdad. El hacedor de verdad debe ser el hecho o estado de cosas consistente en que la gran conjunción es todos los estados de cosas. ${ }^{26}$

Esta presentación de Armstrong no es muy cuidadosa. Por un lado, el hacedor de verdad postulado es un estado de cosas relativo a todos los estados de cosas del mundo; el estado de cosas consistente en que la totalidad de los estados de cosas del mundo es la totalidad de los estados de cosas del mundo. Aquí, sin embargo, aparece un problema grave. O bien lo que Armstrong denomina "la totalidad de los estados de cosas del mundo" incluye el estado de cosas de totalidad, o bien no lo incluye. En la formulación de Armstrong parece que no la incluye. Sin embargo, si no la incluye, entonces el estado de cosas de totalidad es un estado de cosas no contemplado en la totalidad de estados de cosas, de forma que la totalidad en cuestión no puede ser

25 Armstrong da cuenta de la estructura de estos estados de cosas de totalidad. Se trata de estados de cosas relacionales compuestos por la relación $\mathrm{T}$ de totalización y por los siguientes relata: (en este orden) el agregado de estados de cosas y la propiedad de ser un estado de cosas. El estado de cosas resultante puede describirse como el hecho de que el agregado de estados de cosas totaliza la propiedad de ser un estado de cosas. (Armstrong 1997, p. 199.)

Notemos que un estado de cosas así sería el hacedor de verdad de cualquier verdad expresada por un existencial negativo.

26 Armstrong 1997, p. 198. 
tal totalidad. Así, el segundo extremo del dilema tiene consecuencias paradójicas. Debe ser entonces que la totalidad inicial ya contuviera el estado de cosas de totalidad. Pero entonces el argumento de Armstrong que pretende mostrar la necesidad de los estados de cosas de totalidad es simplemente una petición de principio flagrante. Ninguna de ambas alternativas deja a Armstrong en muy buena posición.

Sin embargo, Armstrong parece entender que el primer extremo del dilema, más que generar consecuencias contradictorias, conduce a una regresión infinita:

Existe una pequeña dificultad extraña que deberíamos abordar en este momento. Se trata de la amenaza de una regresión infinita. Si decimos que cierta conjunción de estados de cosas es la totalidad de los estados de cosas, y que esto último es un estado de cosas, entonces parece que la conjunción original no es la totalidad de los estados de cosas. La conjunción original no es más que la totalidad menos el hecho de totalidad. Para introducir este último estado de cosas en la colección debemos acudir a un hecho de totalidad de más alto nivel, y así ad infinitum. En el mejor de los casos, requerimos una infinidad de hechos de este tipo, algo nada atractivo. $^{27}$

Se podría interpretar esta regresión como una salida más airosa al primer extremo del dilema que habíamos planteado, un extremo del que ya hemos dicho que tiene consecuencias contradictorias. Parece evidente, sin embargo, que ni la apelación a una serie infinita de nuevos estados de cosas de totalidad podría mejorar la situación. El problema no es que pueda producirse una regresión infinita, sino que resulta absurdo pensar que si la totalidad original de estados de cosas no resultaba ser una totalidad porque dejaba fuera precisamente el estado de cosas de totalidad postulado por Armstrong (iparadójicamente como un hacedor de verdad de la verdad consistente en que la totalidad original era la totalidad!), la apelación a nuevos estados de cosas de totalidad de órdenes superiores no puede resolver el problema. Al contrario, cada nuevo estado de cosas postulado deviene

27 Id. 
un nuevo "hacedor de falsedad" del enunciado que expresa que la conjunción original de estados de cosas era la totalidad de estados de cosas, enunciado que se presuponía verdadero en el argumento.

En mi opinión, para apreciar el problema con mayor claridad, las afirmaciones de totalidad y sus hacedores de verdad deben relativizarse a diferentes órdenes. Así, debe asignarse a la conjunción original de estados de cosas el orden 0 , y postular como hacedor de verdad del enunciado verdadero que afirma que la conjunción original de estados de cosas de orden 0 es la totalidad de los estados de cosas de orden 0, el estado de cosas de orden 1 que es el estado de cosas de totalidad de los estados de cosas de orden 0. Esto, sin embargo, genera una regresión de hacedores de verdad, porque ahora podemos preguntarnos por el hacedor de verdad del enunciado verdadero que afirma que el estado de cosas de totalidad de los estados de cosas de orden 0 es la totalidad de los estados de cosas de orden 1. Por las razones expuestas para el estadio anterior, el estado de cosas que hace verdadera a esta verdad debe ser de distinto orden; se trata del estado de cosas de orden 2 que es el estado de cosas de totalidad de los estados de cosas de orden 1 . Y así sucesivamente ad infinitum. ${ }^{28}$

En lo que respecta a esta situación generada por la regresión conviene tener claros dos aspectos. El primero es que, en definitiva, no hay ningún estado de cosas de totalidad que fije la clausura fáctica del mundo. El segundo aspecto, relacionado con el primero, es que, de hecho, la situación a la que se llega parece más bien la contraria de la pretendida: ahora tenemos una infinidad de estados de cosas de totalidad añadida a la conjunción original. En lugar de determinar la clausura del mundo,

28 Nótese que también convendría distinguir órdenes para garantizar la consistencia de la construcción Armstrong de los estados de cosas de totalidad (véase la nota 25). Un estado de cosas de totalidad de orden $n$ (para $n>0$ ) se podría construir como el estado de cosas relacional $T_{n}\left(t_{n-1}, E_{n-1}\right)$, donde $T_{n}$ es la relación de totalización de orden $n-1, t_{n-1}$ es el agregado de todos los estados de cosas de orden $n-1$ y $E_{n-1}$ es la propiedad de ser un estado de cosas de orden $n-1$. 
cabría decir que lo hemos expandido. Armstrong se defiende de la regresión del modo siguiente:

Esta dificultad, sin embargo, puede ser abordada a partir de lo que se ha constituido como nuestra respuesta de oficio. La regresión deja de ser amenazadora en el momento en que aparece la superveniencia. El primer hecho de totalidad de estados de cosas es un estado de cosas contingente. Pero los estados de cosas ulteriores a los que hemos apelado después de ese instante son todos ellos implicados por el primer hecho de totalidad. Ésta es la razón por la cual podemos ver que la regresión debe dirigirse al infinito. Puesto que esto es así, podemos decir que los estados de cosas ulteriores supervienen y no involucran ningún incremento ontológico. Podemos conceder que son verdades diferentes. Pero todas esas verdades tienen el mismo hacedor de verdad. El mundo no es más grande por ellos. ${ }^{29}$

En mi opinión esta respuesta entraña dos graves dificultades. En primer lugar, considero que la justificación de Armstrong de la ausencia de incremento ontológico, a partir de la idea de que los términos de alto orden de las regresiones supervienen en los términos de orden más bajo, carece de fundamento. No es obvio en absoluto que la existencia de una relación de dependencia, como la relación de superveniencia, entre una serie de entidades y una entidad, garantice que las primeras no representan ningún incremento ontológico respecto de la segunda, pues una relación de dependencia no es una relación eliminativa.

En segundo lugar, hallamos todavía otro problema concerniente a esta supuesta salida de Armstrong a la regresión que nos ocupa, tal como la hemos planteado con distinciones de orden. Es cierto que el primer hecho de totalidad (el de primer orden) es un hecho contingente, ya que, en principio, nada excluye que pudiera haber más estados de cosas de orden 0. Sin embargo, lo que no resulta evidente es la razón por la cual el resto de hechos

29 Armstrong 1997, pp. 198-199. No deja de resultar notorio que Armstrong parezca quedar satisfecho con su respuesta a las regresiones que se le atribuyen, cuando parece tratarse de una respuesta de orden general exportable, entre otros casos, a las teorías nominalistas de las propiedades que había considerado refutadas, en buena medida, por la fuerza de los argumentos basados en regresiones análogas a las que estamos discutiendo para su teoría. 
de totalidad son necesarios una vez fijados los estados de cosas de orden 0 y el estado de cosas de totalidad de orden 1. Por la misma razón que podría haber habido más estados de cosas de orden 0 , también podría haber habido otros estados de cosas de orden 1, además de otros estados de cosas de totalidad relativos a los estados de cosas de orden 0. En ningún momento se nos dice que no puede haber más estados de cosas de este tipo, y una vez se ha abierto la veda para los estados de cosas de alto orden, ¿qué impide que pueda haber otros hechos de orden 1, como el hecho de que exista un número par (o impar) de estados de cosas de orden 0 ?

\subsection{El argumento de la honda}

En este apartado discutiremos y rebatiremos otra objeción que Oliver presenta a la teoría de los hacedores de verdad de Armstrong. ${ }^{30}$ Se trata de una versión del argumento de la honda de Davidson, aplicado en este caso a los hacedores de verdad. ${ }^{31}$ El argumento pretende concluir que dos enunciados verdaderos cualesquiera (contingentes o no) tienen los mismos hacedores de verdad, a partir de dos supuestos. El primer supuesto, que se deriva de la definición de hacedor de verdad, es que un hacedor de verdad para un enunciado $\mathrm{S}$ también es un hacedor de verdad para cualquier enunciado necesariamente equivalente a S. El segundo supuesto es el siguiente: un hacedor de verdad para $\mathrm{S}$ es un hacedor de verdad para cualquier enunciado que se pueda obtener a partir de $\mathrm{S}$ sustituyendo un término singular del enunciado por otro término singular correferencial. Ahora, consideremos un enunciado verdadero, S, y su hacedor de verdad, T. Por el primer supuesto, T hace verdadero al enunciado ' $\{x: x$ es un número natural y $S\}=\{x: x$ es un número natural $\}$ '. Así, por el segundo supuesto, $\mathrm{T}$ hace verdadero al enunciado ${ }^{\prime}\{x: x$ es un número natural y $\left.S^{*}\right\}=\{x: x$ es un número natural $\}$ ', donde $S^{*}$ es simplemente un enunciado verdadero cualquiera.

30 Oliver 1996, p. 73.

31 Davidson 1984, p. 19. El argumento de Davidson pretende establecer que si los enunciados tienen referentes, entonces todos los enunciados que tienen el mismo valor veritativo tienen los mismos referentes. 
Por el primer supuesto, podemos concluir que $\mathrm{T}$ hace verdadero a $S^{*}$. El argumento, en caso de ser correcto, resultaría devastador para la teoría de los hacedores de verdad, porque ataca a la idea de que al menos para cierto tipo de enunciados verdaderos, seguramente por especificar, existe un hacedor de verdad, o clase mínimamente restringida de hacedores de verdad, que explica su verdad.

Sin embargo, conviene observar que el argumento de Oliver, tal como lo presenta, pone de manifiesto su debilidad. La réplica, más inmediata que en otras aplicaciones del argumento de la honda, es que el segundo supuesto es perfectamente rechazable. Este supuesto pretende pasar por una versión del principio de sustitución de términos singulares correferenciales, extendida, sin embargo, a un contexto en el cual los términos singulares involucrados son mencionados, no usados. Sin embargo, la extensión en cuestión es ilegítima. El problema se hace evidente en la aplicación de este supuesto en el curso del argumento, al pasar de:

T hace verdadero al enunciado ' $\{x: x$ es un número natural y $S\}=\{x: x$ es un número natural $\}$ '

a:

T hace verdadero al enunciado ' $\{x: x$ es un número natural $\left.\mathrm{y} S^{*}\right\}=\{x: x \text { es un número natural }\}^{\prime}$

En efecto, los términos singulares ' $\{x: x$ es un número natural y $S\}$ ' y ' $\left\{x: x\right.$ es un número natural y $\left.S^{*}\right\}$ ' correfieren, pero en el contexto en el que son sustituidos, estos términos son mencionados, no usados, como indica explícitamente el recurso de las comillas.

Por otra parte, aunque existen otras versiones del argumento de la honda cuya respuesta es menos obvia que la que hemos ofrecido para la versión de Oliver, pienso que también deben ser rechazadas. A continuación presentaré una de estas versiones y apuntaré las razones por las que debe ser considerada falaz. Dichas razones han sido expuestas con todo detalle por García-Carpintero y Pérez Otero (1998), y están basadas en las consideraciones de Smullyan (1948). 
Consideremos dos enunciados cualesquiera, ' $\mathrm{S}$ ' y ' $\mathrm{G}$ '. El argumento parte del supuesto con arreglo al cual las verdades de la forma 'El hecho de que $\mathrm{X}$ hace verdadero a ' $\mathrm{S}$ ' ' se preservan:

(i) bajo sustitución de términos singulares correferenciales, y

(ii) bajo sustitución de enunciados lógicamente equivalentes.

Dados estos supuestos, el argumento discurre del siguiente modo:

1. El hecho de que $\mathrm{S}$ hace verdadero a ' $\mathrm{S}$ '.

2. El hecho de que $((7 \mathrm{x})(\mathrm{x}=$ Diogenes $)=((7 \mathrm{x})(\mathrm{x}=$ Diogenes $\&$

S) hace verdadero a ' $\mathrm{S}$ '. (1, (ii))

3. El hecho de que $((7 \mathrm{x})(\mathrm{x}=$ Diogenes $)=((7 \mathrm{x})(\mathrm{x}=$ Diogenes $\&$

G) hace verdadero a ' $\mathrm{S}$ '. (2, (i))

4. El hecho de que $\mathrm{G}$ hace verdadero a ' $\mathrm{S}$ ' $(3,(\mathrm{ii}))$.

Así, se concluye que cualquier hecho hace verdadero a cualquier enunciado verdadero. Sin embargo, como García-Carpintero y Pérez Otero muestran, tanto si tratamos las descripciones definidas al modo de Russell, como si las consideramos como términos singulares genuinamente referenciales, el argumento resulta fallido. Tomemos primero la concepción russelliana de las descripciones. En ese caso, a las descripciones que aparecen en 2 se les debe atribuir menor alcance que al operador 'el hecho de que', si se quiere validar el paso de 1 a 2 . Sin embargo, 3 se sigue de 2 sólo si a las descripciones en 2 y 3 se les atribuye el mayor alcance. Además, si las descripciones son consideradas como términos singulares genuinos, entonces el argumento es una mera petición de principio, pues asume la extensionalidad del operador 'el hecho de que' al aplicar el supuesto (i).

2.5. Los estados de cosas como hacedores de verdad

Para acabar, discutiré la identificación que efectúa Armstrong de los hacedores de verdad con estados de cosas, y trataré de 
argumentar que esta identificación es problemática porque genera una regresión. En el siguiente texto, Armstrong defiende la necesidad de la existencia de un hacedor de verdad para los enunciados predicativos simples e identifica dicho hacedor con un estado de cosas:

Supongamos que el particular $a$ ejemplifica el universal F, $a$ es F. ¿No debería haber algo en el mundo que hiciera que esta verdad sea el caso, que sirva de fundamento ontológico? (Aquí, hacer que sea el caso, por supuesto, no es un hacer que sea el caso causal). El hacedor de verdad, o fundamento, no puede ser $a$, al menos si $a$ es considerado como el particular en sentido estricto, el particular aparte de sus propiedades. ¿Puede ser el par de $a$ y F? Nos vamos aproximando. Sin embargo, lo que parece ser el argumento decisivo contra esta sugerencia es que sería posible que $a$ y $\mathrm{F}$ existiesen ambos $\mathrm{y}$, no obstante, $\mathrm{F}$ podría ejemplificarse en otro lugar.

$[\ldots]$

Estamos preguntando qué cosa del mundo garantizará, hará verdadera, servirá como fundamento ontológico para la verdad de que $a$ es F. El candidato obvio parece ser el estado de cosas consistente en que $a$ es F. En este estado de cosas (hecho, circunstancia) $a$ y F están reunidos. ${ }^{32}$

La cuestión central es cómo debe entenderse esa "reunión" entre el particular y el universal; esto es, cuál es la estructura de los estados de cosas. Armstrong oscila a lo largo de su obra entre adoptar el postulado de la relación de ejemplificación o la hipótesis del vínculo no relacional. Esta oscilación se refleja también en su último libro, al cual pertenece la cita anterior. A mi juicio, la hipótesis del vínculo no relacional debe desecharse

32 Armstrong 1997, pp. 115-116. 
por ininteligible. Sin embargo, como indican Lewis y Oliver, ${ }^{33}$ el otro extremo del dilema genera la vieja regresión de Bradley.

Como respuesta a la objeción de la regresión, Armstrong (1997) sostiene que el enunciado ' $a$ es $F$ ' tiene como hacedor de verdad el estado de cosas $\mathrm{I}(a, \mathrm{~F})$ (donde ' $\mathrm{I}$ ' designa la relación de ejemplificación), y todos los enunciados cuya verdad es garantizada por la regresión de Bradley, como 'a ejemplifica F', 'a y F (en este orden) ejemplifican Ejemplificación,', 'a, F y Ejemplificación $_{1}$ (en este orden) ejemplifican Ejemplificación,', etc., tienen el mismo hacedor de verdad. ${ }^{34}$

No obstante, esta solución no es, a mi jucio, una buena solución. La idea de Armstrong de que una vez fijado el estado de cosas - es decir, una vez fijados el particular $a$, el universal F y la relación de ejemplificación I- quedan fijados todos los otros términos de la regresión no es, a mi juicio, correcta. ${ }^{35}$ Veamos por qué. Por un lado, no es cierto que la existencia del particular y del universal monádico en cuestión garanticen el estado de cosas, ya que un juez discreto y un político honesto no garantizan un juez honesto. Como indica el propio Armstrong, la estructura del estado de cosas que involucra al particular $a$ y al universal F no es mereológica. Ahora, dejando al margen la posibilidad de "vínculos no relacionales", hay que postular la

33 Lewis 1983, pp. 351-352 y Oliver 1996, p. 72. Oliver juzga innecesaria esta apelación de Armstrong a la relación una vez admitidos en su ontología los estados de cosas. Sin embargo, en mi opinión, la presencia de los estados de cosas en la ontología no permite prescindir sin más de la relación de ejemplificación. Puesto que en los estados de cosas el vínculo entre los universales y los particulares no puede ser mereológico, es necesario dar una explicación de la estructura interna de los estados de cosas, de cómo en ellos se conectan sus componentes, universales y particulares. De nuevo tenemos el fatídico dilema. Si el vínculo es relacional, aparece la regresión. Si se pretende que el vínculo no es relacional, debería aclararse algo más su naturaleza de lo que hace Armstrong. Sin embargo, tengo serias dudas acerca de la posibilidad de hacer inteligible esta última noción.

34 Armstrong 1989, pp. 109-110.

35 ¿№ se podría argumentar que el único hacedor de verdad exigido por cada paso en la regresión después del primero (la introducción del vínculo fundamental) no es más que el estado de cosas original? Muchas verdades, si se quiere, pero tan sólo un único hacedor de verdad." Armstrong 1997, pp. 118-119. 
relación de ejemplificación, I, para poder dotar de estructura y unidad al estado de cosas original. Sin embargo, como sucedía con la unión mereológica de $a$ y F, que no garantizaban el estado de cosas consistente en que $a$ es F, la mera existencia de la relación de ejemplificación, I, de $a$ y de $\mathrm{F}$, tampoco garantiza el mencionado estado de cosas: es necesario, no sólo que I exista, sino también que se ejemplifique entre $a \mathrm{y} \mathrm{F}$, y así se inicia de nuevo la regresión. La idea de Armstrong de que podemos fijar el estado de cosas que $a$ es $\mathrm{F}$ fijando $a, \mathrm{~F}$ e I, no es más que una petición de principio.

\subsection{Conclusiones}

Como hemos visto, hay tres tipos de objeciones relativas al uso que hace Armstrong de la noción de hacedor de verdad para argumentar a favor del realismo de universales: las derivadas de la asunción del axioma del hacedor de verdad, el argumento de la honda y la regresión derivada de la identificación de los hacedores de verdad con los estados de cosas. Por lo que respecta a las objeciones del primer grupo, considero que sólo el problema de los existenciales negativos constituye una objeción seria. Como he tratado de mostrar, dicho problema, al igual que su identificación de los hacedores de verdad con estados de cosas, conduce a Armstrong a una regresión. Además, he rechazado que la versión de Oliver del argumento de la honda tenga aplicación en el contexto de los hacedores de verdad y he apuntado razones generales dadas por otros autores que justifican pensar que dicho argumento no constituye amenaza alguna para los hacedores de verdad. $^{36}$

\section{BIBLIOGRAFÍA}

36 Quisiera agradecer a las siguientes personas sus comentarios a trabajos previos que dieron origen a este artículo: Ramón Cirera, José Díez, Manuel García-Carpintero, Andoni Ibarra, Carlos Ulises Moulines y Josep Lluís Prades. La investigación a partir de la cual se ha elaborado este artículo ha sido parcialmente financiada por la DGICYT (PB98-0495-C08-07 y BFF2000-509) y por una Ayuda a Grupos Precompetitivos de la Universitat de Girona. 
Armstrong, D.M., 1997, A World of States of Affairs, Cambridge University Press.

__, 1978, Universals and Scientific Realism, vol. I, Nominalism and Realism, Cambridge University Press.

- , 1978, Universals and Scientific Realism, vol. II, A Theory of Universals, Cambridge University Press.

Davidson, D., 1984, An Inquiry into Truth and Interpretation, Blackwell.

Devitt, M., 1980, “'Ostrich Nominalism’ or 'Mirage Realism'?”, Pacific Philosophical Quaterly, 61, pp. 433-439.

Fox, J.F., 1987, "Truthmaker", Australasian Journal of Philosophy, 65, 2, pp. 188-207.

García-Carpintero, M. y M. Pérez Otero, 1998, "Davidson, Correspondence Truth and the Frege-Gödel-Church Argument", History and Philosophy of Logic, 19, pp. 63-81.

Lewis, D., 1992, "Critical Notice of D.M. Armstrong's A Combinatorial Theory of Possibility", Australasian Journal of Philosophy, 70, 2, pp. 211-224.

, 1983, "New Work for a Theory of Universals", Australasian Journal of Philosophy, 61, 4, pp. 343-377.

Mortensen, C., 1987, "Arguing for Universals", Revue Internationale de Philosophie, 41, 160, pp. 97-111.

Mulligan, K., P. Simons y B. Smith, 1984, "Truth-Makers", Philosophy and Phenomenological Research, 44, 3, pp. 287-321.

Oliver, A., 1996, "The Metaphysics of Properties", Mind, vol. 105, no. 417, pp. 1-80.

Smullyan, A., 1948, "Modality and Description", Journal of Symbolic Logic, 13, pp. 31-37.

Van Cleve, J., 1994, "Predication Without Universals? A Fling with Ostrich Nominalism", Philosophy and Phenomenological Research, 54, 3, pp. 577-590. 